\title{
Gold Nanoparticles Increase PLK1-Specific Small Interfering RNA Transfection and Induce Apoptosis of Drug Resistance Breast Cancer Cells
}

\author{
Ying Tian,, ${ }^{1}$ Yunlei Zhang, ${ }^{1}$ Jing Pan, ${ }^{1}$ Nan Lu, ${ }^{1}$ Shouju Wang, ${ }^{1}$ and Guangming Lu ${ }^{1,2}$ \\ ${ }^{1}$ Department of Medical Imaging, Jinling Hospital, School of Medicine, Nanjing University, Nanjing 210002, China \\ ${ }^{2}$ State Key Laboratory of Analytical Chemistry for Life Science, School of Chemistry and Chemical Engineering, \\ Nanjing University, Nanjing 210093, China
}

Correspondence should be addressed to Shouju Wang; shouju.wang@gmail.com and Guangming Lu; cjr.luguangming@vip.163.com

Received 22 May 2015; Accepted 27 July 2015

Academic Editor: Anh-Tuan Le

Copyright (C) 2015 Ying Tian et al. This is an open access article distributed under the Creative Commons Attribution License, which permits unrestricted use, distribution, and reproduction in any medium, provided the original work is properly cited.

\begin{abstract}
Drug resistance is a major barrier that limits the effectiveness of chemotherapies against breast cancer. Here, gold nanoparticles (GNPs) characterized by good dispersivity, high stability, low cytotoxicity, and simple synthesis were developed to deliver small interfering RNA (siRNA) against PLK1 (PLK1-siRNA) and overcome the drug resistance of breast cancer cells. Compared with the commonly used Lipofectamine 2000, GNPs showed higher PLK1-siRNA delivery efficiency and resulted in the remarkable gene silencing of PLK1 in drug resistance breast cancer cells MCF-7/MDR1 with low cytotoxicity in vitro. Moreover, delivery of PLK1siRNA by GNPs could cause $14.23 \%$ apoptosis of MCF-7/MDR1 cells, which was apparently higher than $11.01 \%$ apoptosis conducted by Lipofectamine 2000. In addition, GNPs showed strong X-ray attenuation coefficient, indicating the potential theranostic application of this system. Therefore, this study disclosed an important step in the use of GNPs as transfection vector of siRNA that will be of great benefit to gene therapy against drug resistant cancer.
\end{abstract}

\section{Introduction}

Breast cancer is the most common cancer in women worldwide which is expected to account for $29 \%$ of all new cancers among women in the near future $[1,2]$. The currently used clinical methods in cancer treatment mainly include surgery, chemotherapy, radiation therapy, immunotherapy, and biologic therapy [3]. Efficient suppression of growth of tumors and their metastatic lesions is a vital factor for breast cancer therapy [4]. However, despite the remarkable progress in breast cancer treatment in the last decade, the appearance of drug resistance greatly decreases the prognosis and survival rate of cancers and leads to $\sim 90 \%$ failure of chemotherapy treatment in patients with metastatic cancers $[5,6]$. Therefore, new strategies to overcome the drug resistance of breast cancer and increase antitumor efficacy are urgently needed.

The most prominent gene involved in drug resistance is multidrug-resistant protein 1 (MDR1), encoding the drug efflux transporter P-glycoprotein. The overexpression of MDR1 makes cancer cells resistant to a wide range of chemotherapeutic drugs $[7,8]$. Previous studies showed that the overexpression of oncogene polo-like kinase 1 ( $P L K 1$ ) could upregulate the transcriptional expression of activator protein 1, one of the most important transcription factors of DNA binding protein, resulting in elevation of its target MDR1 [9]. PLK1, a serine/threonine protein kinase, plays a key role in cell proliferation. Its overexpression is closely related to poor prognosis in cancer treatment $[10,11]$. Knockdown of PLK1 gene by small interfering RNA (siRNA) could downregulate the expression of MDR1 and induce apoptosis of cancer cells. This will greatly benefit the treatment of drug resistant breast cancers [12].

RNA interference (RNAi) as one of the most powerful methods for gene knockdown has shown its great potential application in cancer gene therapy $[13,14]$. However, siRNA by itself is difficult to exert silencing function due to its 
fast degradation in physiological environment and poor cell penetrability. A well-designed vector will greatly increase the therapeutic efficiency of siRNA [15]. Currently, kinds of vectors including liposomes, polymers, and peptides have been developed [16-18]. These vectors can protect siRNA from degradation and improve delivery efficiency. However, the clinical applications of these methods are hindered by their complicated procedure, potential toxicity, and inability for theranostic applications. Therefore, exploring new delivery vectors with the promising advantages will be greatly valuable to gene therapy [19-22].

Gold nanoparticles (GNPs) are featured with small size, good dispersivity, high stability, low cytotoxicity, and simple synthesis procedure [23, 24]. They have been proved as potential candidates in biomedical application. The surface of GNPs can be easily modified by oligonuclear acids, large biomolecules, or peptides to boost their cell uptake and facilitate drug-delivery [25]. Furthermore, gold has high atomic number and strong X-ray attenuation coefficient, making it favorable for developing X-ray imaging [26-28]. So, GNPs can be used as the agent for X-ray imaging and siRNA delivery. In this study, polyethylenimine (PEI) coated GNPs were used to transport the specific siRNA against PLK1 (PLK1-siRNA) to conquer drug resistance of MCF-7/MDR1 breast cancer cells.

The in vitro experiments showed that the GNPs could effectively transfer PLK1-siRNA into MCF-7/MDR1 cells with little toxicity. The expression of PLK1 was downregulated after siRNA transfection when the weight ratio between GNPs and siRNA is 4 , evaluated by western blot analysis. The terminal deoxynucleotidyl transferase-mediated dUTP nick end labeling (TUNEL) and flow cytometry assays further demonstrated that GNPs-siRNA transfection led to higher efficiency of apoptosis in MCF-7/MDR1 cells, compared with Lipofectamine 2000. The enhanced X-ray attenuation intensities of GNPs-PEI were also confirmed by X-ray imaging, and this effect promptly increased with the rise of the concentration of gold. This work will be very valuable to further exploit the transfection methods of siRNA, promote gene therapy, and develop biological imaging for drug resistant cancers.

\section{Materials and Methods}

2.1. Materials. Polyethyleneimine (PEI, branched, Mw $25000)$, 11-mercaptoundecanoic acid (MUA), chloroauric acid $\left(\mathrm{HAuCl}_{4} \cdot 3 \mathrm{H}_{2} \mathrm{O}\right)$, and 3-(4,5-dimethylthiazol-2-yl)-2,5diphenyltetrazolium bromide (MTT) were obtained from Sigma-Aldrich. Diethylpyrocarbonate (DEPC) water and Lipofectamine 2000 transfection kit were purchased from Invitrogen. Sodium citrate and dimethyl sulfoxide (DMSO) were got from the Shanghai Chemical Corp. (Shanghai, China). Paraformaldehyde and DAPI were provided by KeyGEN Biotech Co., Ltd. (Jiangsu, China). GelRed was purchased from Biotium Inc. (Heyward, California, USA). Anti-PLK1 antibody and anti-MDR1 antibody were obtained from Abcam Company (Cambridge, UK). Bovine serum albumin (BSA) was got from Amresco Company (Solon, Ohio, USA). In situ cell death detection kit was purchased from Roche Inc. (Indianapolis, Indiana, USA). Human breast cancer cells (MCF-7) and human embryonic kidney (293T) cells were purchased from ATCC (Manassas, VA). Dulbecco's Modified Eagle Medium (DMEM), Opti-MEN I Reduced-Serum Medium (Opti-MEM), fetal bovine serum (FBS), trypsin, and penicillin/streptomycin were bought from Gibco/Life Technologies (Grand Island, New York, USA). The duplexed PLK1-siRNA with or without a Cy 5 fluorophore on the $5^{\prime}$ end of the sense strand was purchased from Ribobio Co., Ltd. (Guangzhou, China).

2.2. Synthesis of GNPs-PEI. The GNPs seed solution was prepared by adding $3 \mathrm{~mL}$ of $1 \%$ sodium citrate solution to $100 \mathrm{~mL}$ of boiling $1.0 \mathrm{mM} \mathrm{HAuCl}_{4}$ solution under vigorous stirring until the transparent solution changed to dark red. The solution cooled to room temperature (RT) before use. Next, $20 \mu \mathrm{L}$ of $20 \mathrm{mM}$ MUA was added to $10 \mathrm{~mL}$ GNPs solution and kept stirring for $1 \mathrm{~h}$ at RT. Then the GNPs were purified by centrifugation for two times and dispersed in $10 \mathrm{~mL}$ water. The aqueous solution was mixed slowly with $1 \mathrm{~mL}$ of $10 \mathrm{mg} / \mathrm{mL}$ PEI and stirred at RT for $2 \mathrm{~h}$. After stirring, the GNPs-PEI solution was adjusted to $\mathrm{pH} 7.0$ and centrifuged for two times to purify before use.

2.3. Cell Culture. Human breast cancer cells (MCF-7), human embryonic kidney cells (293T), and drug resistant breast cancer cells (MCF-7/MDR1) were all cultured in DMEM with $10 \% \mathrm{FBS}$ and $1 \%$ penicillin/streptomycin in a humidified incubator at $37^{\circ} \mathrm{C}$ under $5 \% \mathrm{CO}_{2}$ atmosphere with $95 \%$ relative humidity. The drug resistant cell line MCF-7/MDR1 was established from MCF-7 cells through doxorubicin selection.

2.4. Preparation of PLK1-siRNA. The inhibitory effect of siRNA on the expression of PLK1 gene was tested. The sequence of PLK-siRNA were $5^{\prime}$-UGAAGAAGAUCACCCUCCUUA-3' ${ }^{\prime}, 5^{\prime}$-GGCAACCAAAGUCGAAUAU-3', $5^{\prime}$-GUCUCAAGGCCUCCUAAUA-3', and $5^{\prime}$-GAUCACCCUCCUUAAAUAU-3'. Firstly, MCF-7/MDR1 cells were cultured $\left(2.5 \times 10^{6}\right.$ cells per well $)$ on a 6 -well plate until the confluence reached $\sim 90 \%$. Lipofectamine $2000(10 \mu \mathrm{L})$ was diluted in $250 \mu \mathrm{L}$ Opti-MEM and incubated for $5 \mathrm{~min}$ and then was mixed with $4 \mu \mathrm{g}$ PLK1-siRNA diluted in $250 \mu \mathrm{L}$ Opti-MEM for $30 \mathrm{~min}$ at room temperature. After $6 \mathrm{~h}$ of incubation, the transfection solution was discarded and replaced with fresh DMEM for another $24 \mathrm{~h}$. Cell apoptosis was determined by the in situ cell death detection kit according to the manufacturer's instruction. Images of cell apoptosis under the effect of PLK1-siRNA were recorded by a fluorescence microscope (Olympus IX71, Japan) equipped with fluorescent filter sets (excitation, 450-500 nm; emission, 515-565 nm).

2.5. PLK1-siRNA Binding to GNPs-PEI. GNPs-PEI was mixed with siRNA $(20 \mathrm{mM})$ and incubated for $15 \mathrm{~min}$ at room temperature. To evaluate the siRNA binding ability, various weight ratios between GNPs and siRNA (0 to 4) were mixed and visualized on $1 \%(\mathrm{w} / \mathrm{v})$ agarose gel containing $0.01 \%(\mathrm{v} / \mathrm{v})$ GelRed. The electrophoresis was carried out for $15 \mathrm{~min}$ at a constant voltage of $100 \mathrm{~V}$ in buffer. The siRNA bands were 
differentiated using a Tanon UV Gel Image System (Shanghai, China).

2.6. Characterization of GNPs, GNPs-PEI, and GNPs-siRNA. The sizes and morphologies of the GNPs were characterized by transmission electron microscopy (TEM) using a JEOL JEM-2100 microscope (Japan) at $200 \mathrm{kV}$. The absorbance spectra of GNPs, GNPs-PEI, and GNPs-siRNA were recorded through a UV/VIS spectrometer Lambda 35 (PerkinElmer, USA). The surface charge and the diameter of the samples in water were obtained via a ZetaPALS Analyzer (Brookhaven, USA). About $1 \mathrm{~mL}$ aqueous solution of GNPs-PEI with different weight (35-280 $\mu \mathrm{g})$ was prepared for X-ray imaging through an IVIS Lumina XR (Xenogen Corporation-Caliper, Alameda, CA, USA). And the X-ray intensity ratio between the signal area of GNPs-PEI and the background area was calculated by analysis software.

2.7. Cytotoxicity Assay. MTT assay was employed to test the cytotoxicity of GNPs-PEI against breast cancer cells and normal cells. MCF-7/MDR1 breast cancer cells and 293T normal cells were seeded $\left(1 \times 10^{4}\right.$ cells per well) in a 96well plate, respectively, until the confluence reached $\sim 70 \%$. Then the cells were incubated with different weights of GNPs $(0-1.6 \mu \mathrm{g})$ for $24 \mathrm{~h}$ and $48 \mathrm{~h}$ at $37^{\circ} \mathrm{C}$. Next, the medium was replaced by $200 \mu \mathrm{L}$ medium with $20 \mu \mathrm{L}$ MTT $(5 \mathrm{mg} / \mathrm{mL})$ solution. The cells were incubated for additional $4 \mathrm{~h}$ and diluted in $150 \mu \mathrm{L}$ dimethyl sulfoxide (DMSO) to dissolve the blue formazan crystals. The absorbance at $570 \mathrm{~nm}$ for each well was recorded by an automated microplate reader (BioTek, Winooski, Vermont, USA) to calculate the viability of cells.

2.8. Cellular Uptake Test. Breast cancer cells MCF-7/MDR1 were cultured $\left(5 \times 10^{4}\right.$ cells per well) in a 24 -well plate with cover slips and incubated at $37^{\circ} \mathrm{C}$ with $5 \% \mathrm{CO}_{2}$ for $24 \mathrm{~h}$. The cells on the cover slips were incubated with Lipofectamine 2000 bearing Cy 5 fluorescence-labeling siRNA for $4 \mathrm{~h}$ and $24 \mathrm{~h}$. As parallel groups, the cells were treated with GNPssiRNA (weight ratio $=1$ ) for $4 \mathrm{~h}$ and $24 \mathrm{~h}$. Finally, MCF7/MDR1 cells were washed and fixed in $4 \%$ paraformaldehyde and then stained with DAPI for nucleus imaging. After washing again, the cover slips were mounted and observed using an Olympus Fluoview FV10i (Olympus Instruments, Tokyo, Japan).

2.9. Western Blot Analysis. MCF-7 and MCF-7/MDR1 cells were transfected with PLK1-siRNA using Lipofectamine 2000 and GNPs delivery vehicles as above described. After that, the cells were lysed on ice and centrifuged at $4^{\circ} \mathrm{C}$ for $5 \mathrm{~min}$. About $10 \mu \mathrm{L}$ of the supernatants was used for protein quantitative analysis through Bradford assay (Bio-Rad, USA). The remaining mixtures were mixed with Laemmli's buffer and held at $95^{\circ} \mathrm{C}$ for $10 \mathrm{~min}$ for protein denaturation. Next, after separating proteins in the SDS-PAGE gel, they were electroblotted to a hydrophobic polyvinylidene difluoride (PVDF) membrane, and the membranes were blocked in buffer containing $5 \%(\mathrm{w} / \mathrm{v})$ nonfat dry milk. After washing, membranes were incubated with primary antibody PLK1 $(1: 1000)$ and GAPDH $(1: 1000)$ overnight at $4^{\circ} \mathrm{C}$, respectively, followed by washing and incubation with secondary antibody for $1 \mathrm{~h}$ at RT. The signals of the membrane were recorded by $\mathrm{DAB}$ coloration after washing again. Finally, immunoreactive bands were visualized and quantified using ImageJ software.

2.10. Cell Apoptosis Assay. The PLK-siRNA mediated apoptosis of MCF-7/MDR1 cells was tested by the in situ cell death detection kit of Roche and flow cytometry (FCM) after siRNA transfection using Lipofectamine 2000 or GNPs delivery vehicles. Firstly, MCF-7/MDR1 cells were cultured $\left(2.5 \times 10^{6}\right.$ cells per well) on a 6-well plate until the confluence reached $\sim 90 \%$. The mixtures of Lipofectamine $2000(10 \mu \mathrm{L})$ with PLK1-siRNA $(4 \mu \mathrm{g})$ and GNPs $(4 \mu \mathrm{g})$ carrying PLK1-siRNA $(4 \mu \mathrm{g})$ were added into wells and incubated for $24 \mathrm{~h}$. Cells without any treatment were used as control. Subsequently, the cells were harvested and stained with annexin V/FITC and PI according to the instructions. The apoptotic cells were recorded by a fluorescence microscope and a FACSCalibur flow cytometer (Becton, Dickinson and Company, Franklin Lakes, New Jersey, USA).

2.11. Statistical Analysis. All statistical comparisons were performed using Student's $t$-test. Data was expressed as mean \pm SD. A two-sided $P$ value of less than 0.05 was considered statistically significant in all of the statistical tests.

\section{Results and Discussion}

3.1. PLK1-Specific siRNA Induced Apoptosis of MCF-7/MDR1 Cells. Four different specific siRNAs were chosen to silence PLK1 expression through the widely used Lipofectamine 2000. The possible apoptosis of the treated cells was analyzed by TUNEL assay. As shown in Figure 1, annexin V/FITC labeling MCF-7/MDR1 cells exhibited strong green color after transfection by the Lipofectamine 2000 carrying PLK1siRNA. This demonstrated that the PLK1-siRNA could effectively induce apoptosis of MCF-7/MDR1 cells. Moreover, cells after treatment by siRNA- 4 showed more apoptotic cells than other siRNAs. Therefore, PLK1-specific siRNA- 4 was a perfect candidate to evaluate the transfection efficiency of GNPs.

3.2. Morphology and Characteristics of GNPs, GNPs-PEI, and GNPs-siRNA. Polyethyleneimine (PEI) has been known to be a gene delivery vector because of its high buffering capacity to enhance the endosome escape of nucleic acid. In this study, PEI was functionalized with GNPs to deliver siRNA, which could condense and protect RNA from high cellular internalization. The binding capability of siRNA to GNPs is a key factor to silence PLK1 gene in MCF-7/MDR1 cells. Therefore, different weight ratios between GNPs and siRNA were evaluated by gel retardation assay. As shown in Figure 2(a), the migration of siRNA was gradually retarded as the weigh ratios between GNPs and siRNA increased, compared with free siRNA. The band completely disappeared from the gel when the weight ratio became $1: 1$. This weight ratio was chosen for the following characterization of GNPs. 


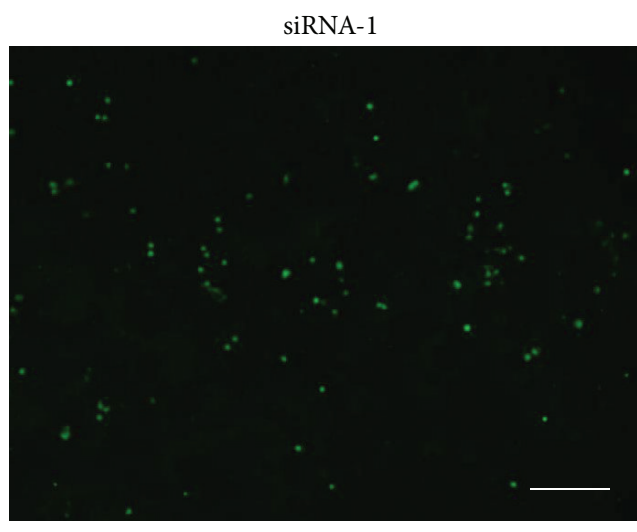

siRNA-3

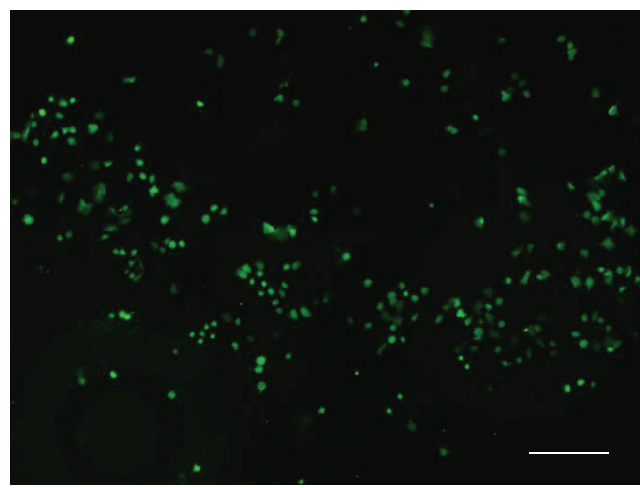

siRNA-2

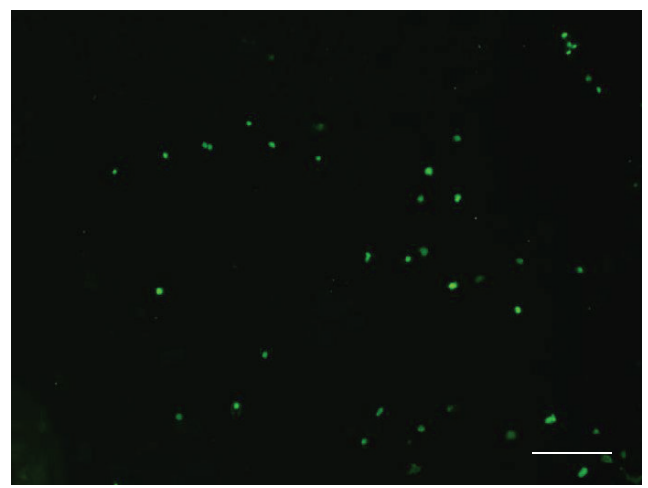

siRNA-4

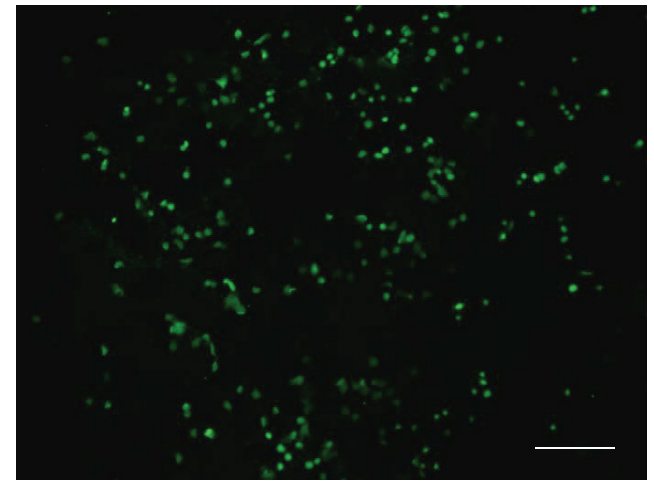

FIGURE 1: PLK1-specific siRNAs induced apoptosis of MCF-7/MDR1 cells. Cells were incubated with four different Lipofectamine 2000siRNAs for $24 \mathrm{~h}$ and evaluated by TUNEL assay. Scale bars: $100 \mu \mathrm{m}$.

This also indicated the high binding efficiency of siRNA to GNPs. The GNPs would be an ideal carrier for RNA delivery.

The morphologies and sizes of GNPs were observed by TEM. Figure 2(b) showed that GNPs exhibited excellent dispersivity and spherical structure with an average diameter of $\sim 20 \mathrm{~nm}$. As shown in Figure 2(c), GNPs, GNPs-PEI, and GNPs-siRNA displayed broad plasmon spectra peaked at $\sim 520 \mathrm{~nm}$ due to the surface plasmon resonance (SPR) of gold, which indicated that siRNA was successfully loaded onto the surface of gold under the assistance of PEI but slightly influenced the absorbance value of particles. The zeta potential of the nanoparticles exhibited positive charge at $36.70 \pm 3.00 \mathrm{mV}$ and $38.12 \pm 2.23 \mathrm{mV}$, due to the PEI modification, but showed negative charge at $-34.33 \pm 1.03 \mathrm{mV}$ before modification (Figure 2(d)). The dynamic light scattering (DLS) assay demonstrated that the hydrodynamic sizes of GNPs-PEI and GNPs-siRNA were $52.7 \pm 0.7 \mathrm{~nm}$ and 54.3 $\pm 0.7 \mathrm{~nm}$, respectively, which were higher than unmodified GNPs $(29.7 \pm 0.7)$ (Figure 2(e)).

Gold has large atomic number and strong X-ray attenuation coefficient, making it favorable for X-ray imaging. Figure 2(f) showed that the X-ray attenuation intensities of GNPs-PEI increased as the rise of gold concentrations. At higher concentration $(280 \mu \mathrm{g})$, the X-ray intensity ratio of GNPs-PEI could exceed 4 . The results revealed that
GNPs-PEI could strengthen the X-ray attenuation intensities in a concentration-dependent way, indicating the potential theranostic application of this siRNA delivery system.

\subsection{Cytotoxicity of GNPs against MCF-7/MDR1 Cancer Cells} and 293T Normal Cells. The toxicity of nanoparticles is a vital factor for their clinical translation application. Here, the biocompatibility of GNPs-PEI was determined by treating MCF-7/MDR1 breast cancer cells and 293T normal cells with different weights of GNPs-PEI. The MTT results showed that the weight of gold (from $0.1 \mu \mathrm{g}$ to $1.6 \mu \mathrm{g}$ ) did not affect the proliferation of the cells. The viability of cancer cells and normal cells totally surpassed $90 \%$ after $24 \mathrm{~h}$ and $48 \mathrm{~h}$ incubation with GNPs-PEI (Figure 3). This indicated that the PEI-modified gold nanoparticles were not toxic to both cancer and normal cells and exhibited well biocompatibility.

3.4. Cellular Uptake Study. Confocal laser scanning microscopy was used to evaluate the transfection of siRNA into cells conducted by Lipofectamine 2000/GNPs after incubation for $4 \mathrm{~h}$ and $24 \mathrm{~h}$. It was observed that the GNPssiRNA labeled with Cy 5 (red) entered MCF-7/MDR1 cells and was distributed in cytoplasm around the nucleus after $4 \mathrm{~h}$ treatment, but not Lipofectamine 2000-siRNA 


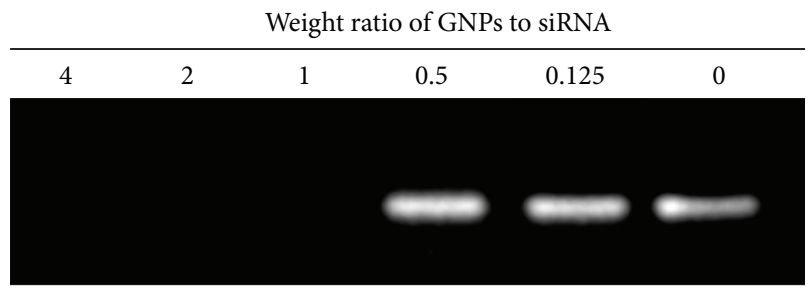

(a)

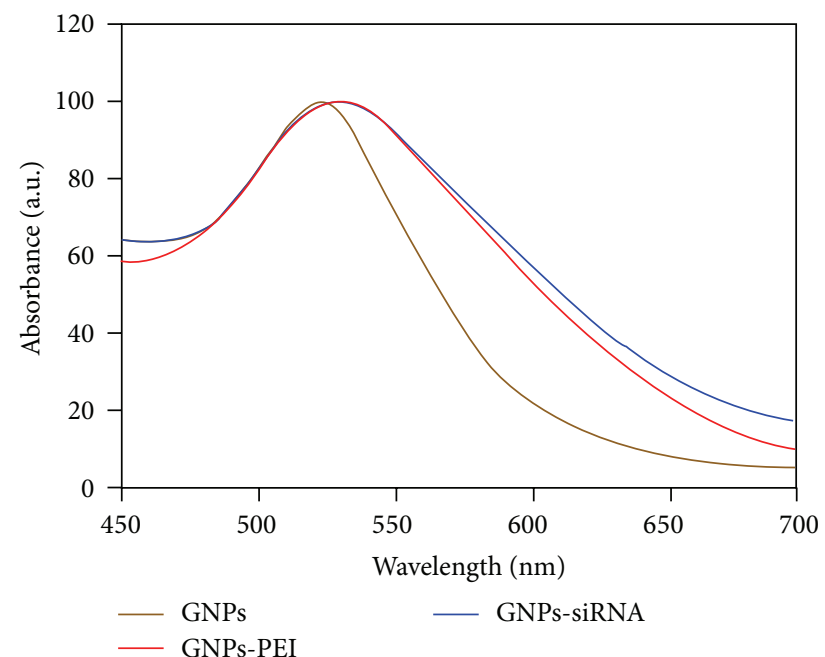

(c)

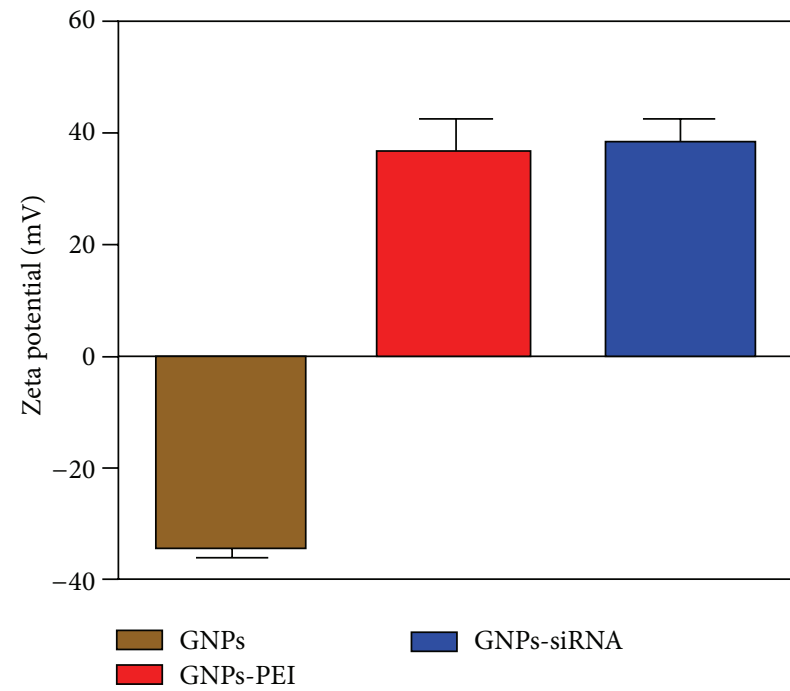

(e)
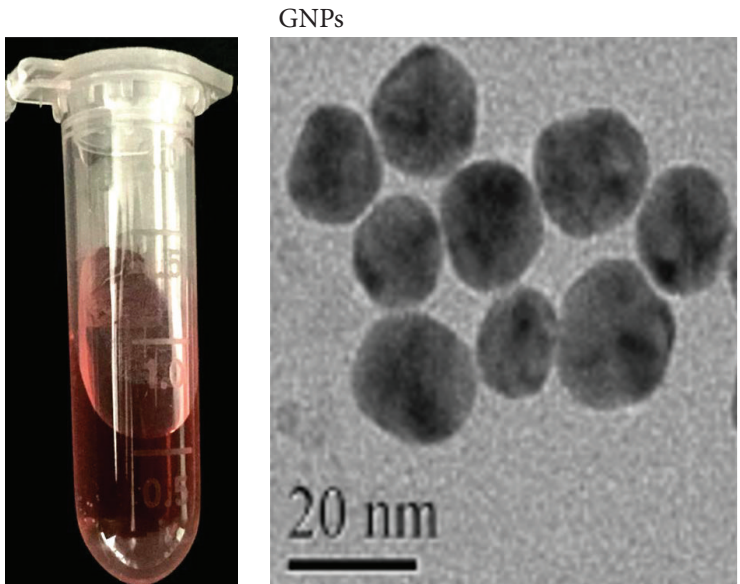

(b)

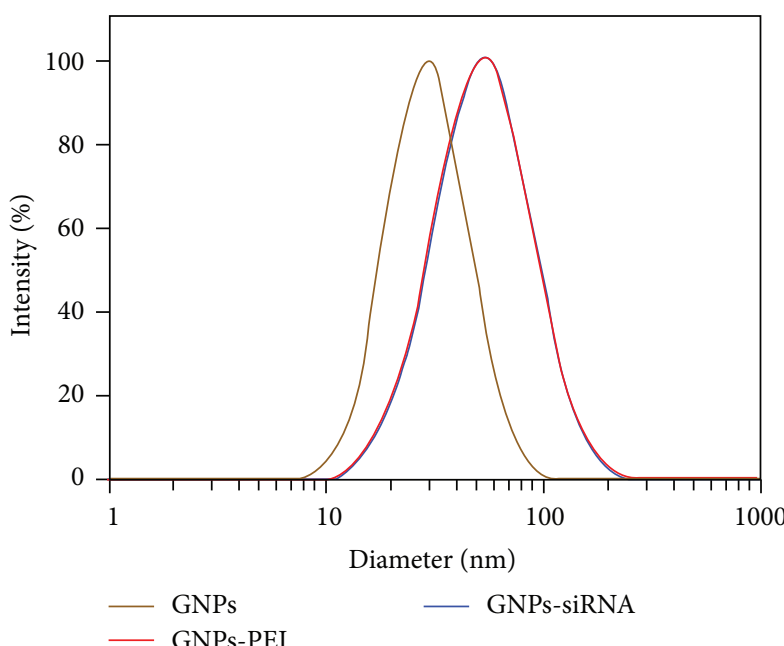

(d)

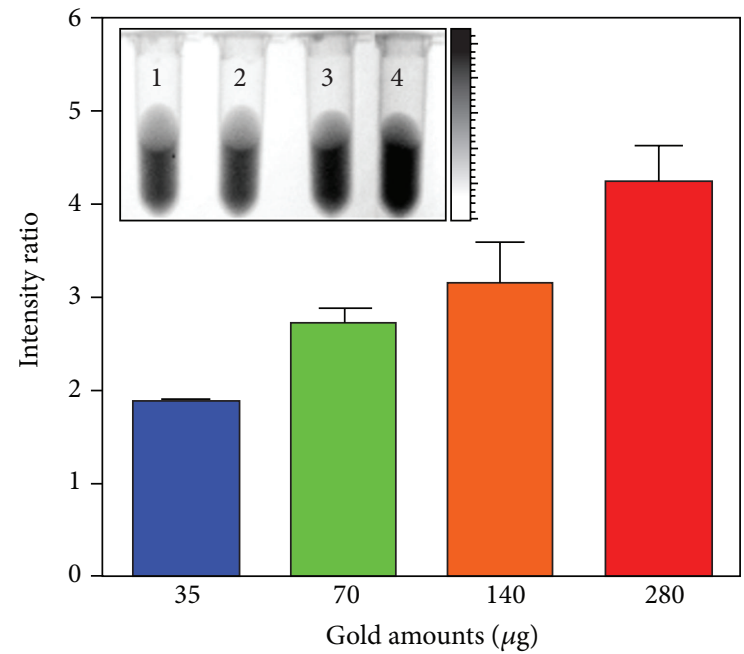

(f)

Figure 2: (a) Gel retardation assay of GNPs-siRNA. Lanes from left to right indicate different weight ratios $(4,2,1,0.5$, and 0.125$)$ between GNPs and siRNA. The 0 weight ratio stands for free siRNA. (b) TEM images of GNPs. Scale bars: $20 \mathrm{~nm}$. (c) UV-VIS spectra of GNPs, GNPsPEI, and GNPs-siRNA from 450 to $700 \mathrm{~nm}$. (d) Zeta potential of GNPs, GNPs-PEI, and GNPs-siRNA. (e) Diameters of GNPs, GNPs-PEI, and GNPs-siRNA. (f) X-ray images and intensity ratios of GNPs-PEI with different weights (1: $35 \mu \mathrm{g}, 2: 70 \mu \mathrm{g}, 3: 140 \mu \mathrm{g}$, and 4: $280 \mu \mathrm{g}$ ). 

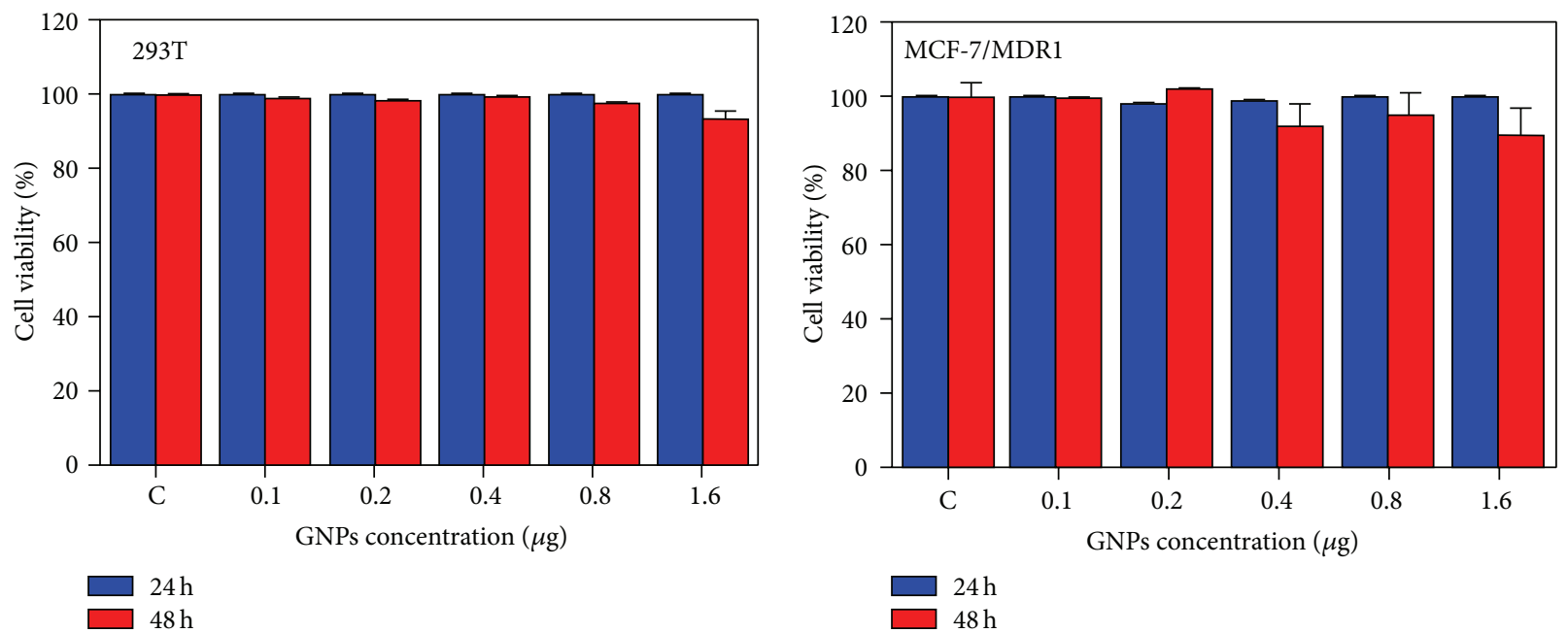

FIgURE 3: Cell viability of 293T cells and MCF-7/MDR1 cells after incubation with GNPs-PEI at different weights for $24 \mathrm{~h}$ and $48 \mathrm{~h}$.

(Figure 4). At $24 \mathrm{~h}$, the GNPs-siRNA treated group showed higher red fluorescence intensities, and the intensity of GNPs-siRNA treated group was conspicuously stronger than Lipofectamine 2000-siRNA group. The result clearly demonstrated that the transfection of siRNA conducted by GNPs was in a time-dependent manner and showed higher efficiency than the commonly used Lipofectamine 2000. This indicated that GNPs-based RNA delivery system had potential to be used in gene therapy.

3.5. GNPs-siRNA Transfection Restrained the Expression of $P L K 1$ Gene. To further investigate the knockdown efficiency of GNPs-siRNA, the expression of the siRNA targeting genePLK1 was analyzed by western blot. The high expression of PLK1 protein generally concerns the proliferation of malignant cells. The results showed that MCF-7/MDR1 drug resistant breast cancer cells had more PLK1 protein than MCF7 cancer cells (Figure 5). Compared with the control, there was almost no change of PLK1 band in GNPs-treated group. However, the protein level of PLK1 obviously decreased in the cells after treatment by Lip-siRNA and GNPs-siRNA at different weights, especially the weight of 4 between GNPs and siRNA. The amount of PLK1 protein in MCF7/MDR1 cells reduced almost $49 \%$ and $81 \%$ after treatment with Lipofectamine and GNPs, respectively (Figure 5). This indicated that GNPs-mediated transfection of siRNA resulted in significant inhibition of PLK1 gene expression in MCF7/MDR1 cells, and it was more efficient than Lipofectamine 2000. The results indicated that GNPs-siRNA was suitable for the delivery of siRNA and could efficiently downregulate its targeting genes.

3.6. GNPs-siRNA Transfection Induced Apoptosis of MCF7/MDR1 Cells. PLK1 is closely related to cell division and apoptosis. The downregulation of PLK1 could induce apoptosis of drug resistance cells. Therefore, TUNEL assay and flow cytometry were employed to evaluate the apoptosis of MCF-7/MDR1 cells after treatment by GNPs-siRNA. In TUNEL assay, both GNPs-siRNA and Lip-siRNA resulted in apoptosis of MCF-7/MDR1 cells, compared with the control group (Figure 6(a)). However, GNPs-siRNA treated group showed more apoptotic cells than Lip-siRNA by comparing the green fluorescence images (apoptotic cells) with the images (total cells) taken under the bright field. The flow cytometry test further confirmed that GNPs-siRNA and LipsiRNA could induce apoptosis of MCF-7/MDR1 cells, and the apoptosis rates got to $14.23 \%$ and $11.01 \%$ after $24 \mathrm{~h}$ incubation, respectively, while there was only $1.71 \%$ apoptosis of cells in the control group (Figure 6(b)). Apoptosis rate was closely related to the amounts of PLK1-siRNA in the cells. This meant that GNPs could deliver more PLK1-specific siRNA into the cells than Lipofectamine 2000. The assay provided convincing evidence that GNPs-siRNA was a favorable vector for siRNA delivery and would greatly benefit the gene therapy for cancer.

\section{Conclusions}

In this study, PEI-capped GNPs efficiently delivered PLK1specific siRNA into the drug resistance MCF-7/MDR1 breast cancer cells and showed higher transfection efficiency than the commercial kit Lipofectamine 2000. The transfection of PLK1-specific siRNA into cells not only silenced its targeting genes but also induced apoptosis of the drug resistant breast cancer cells. It was worth noting that exclusive GNPs were not toxic to normal/cancer cells. Importantly, the GNPs could be visualized by X-ray imaging in a concentration-dependent manner because of the excellent properties of gold. Overall, this work disclosed the great potential of GNPs as the excellent delivery system in gene therapy for drug resistance cancers and the further application of X-ray imaging. 

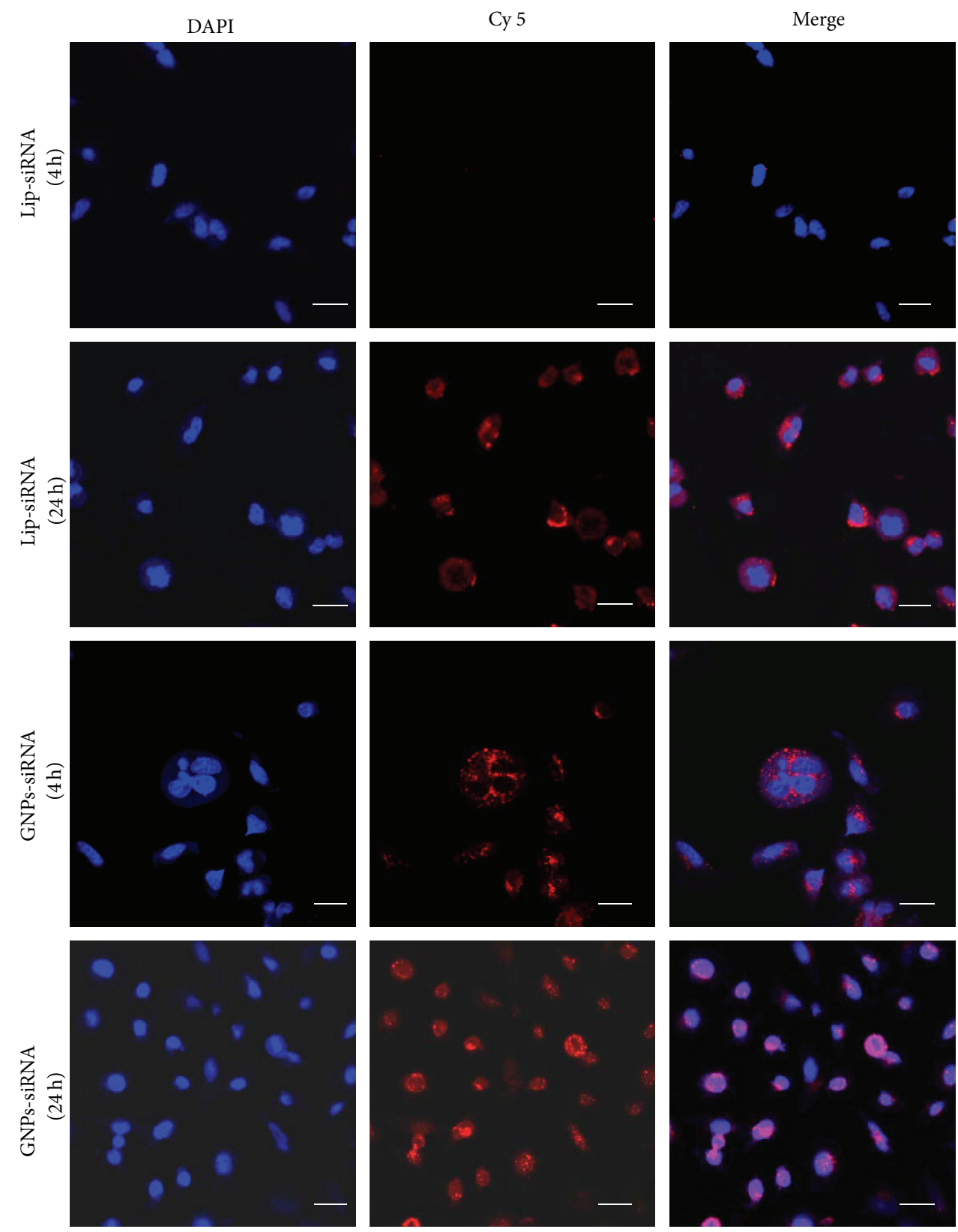

FIGURE 4: Internalization of Lipofectamine 2000-siRNA (Lip-siRNA) and GNPs-siRNA (the weight ratio was 1) by MCF-7/MDR1 cells after $4 \mathrm{~h}$ and $24 \mathrm{~h}$ incubation. DAPI (blue) stains cell nuclei and Cy 5 (red) indicates siRNA. Scale bars: $20 \mu \mathrm{m}$.

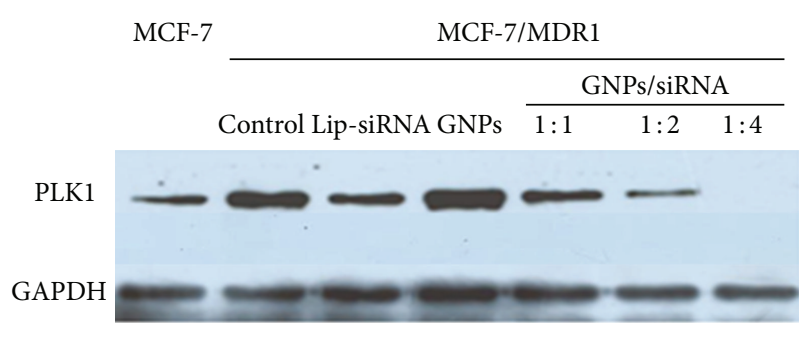

FIGURE 5: Western blot analysis of PLK1 expression in MCF-7/MDR1 and MCF-7 cells. MCF-7/MDR1 and MCF-7 cells were incubated with Lipofectamine 2000-siRNA (Lip-siRNA), GNPs alone, and GNPs-siRNA with different weight radios for $24 \mathrm{~h}$.

\section{Conflict of Interests}

The authors declare that there is no conflict of interests regarding the publication of this paper.

\section{Acknowledgments}

This project was financially supported by the National Key Basic Research Program of China (2014CB744504), the Major International (Regional) Joint Research Program of China (81120108013), the National Natural Science Foundation of China (81201175, 81371611, 81471632, 81401469, and U1332117), 

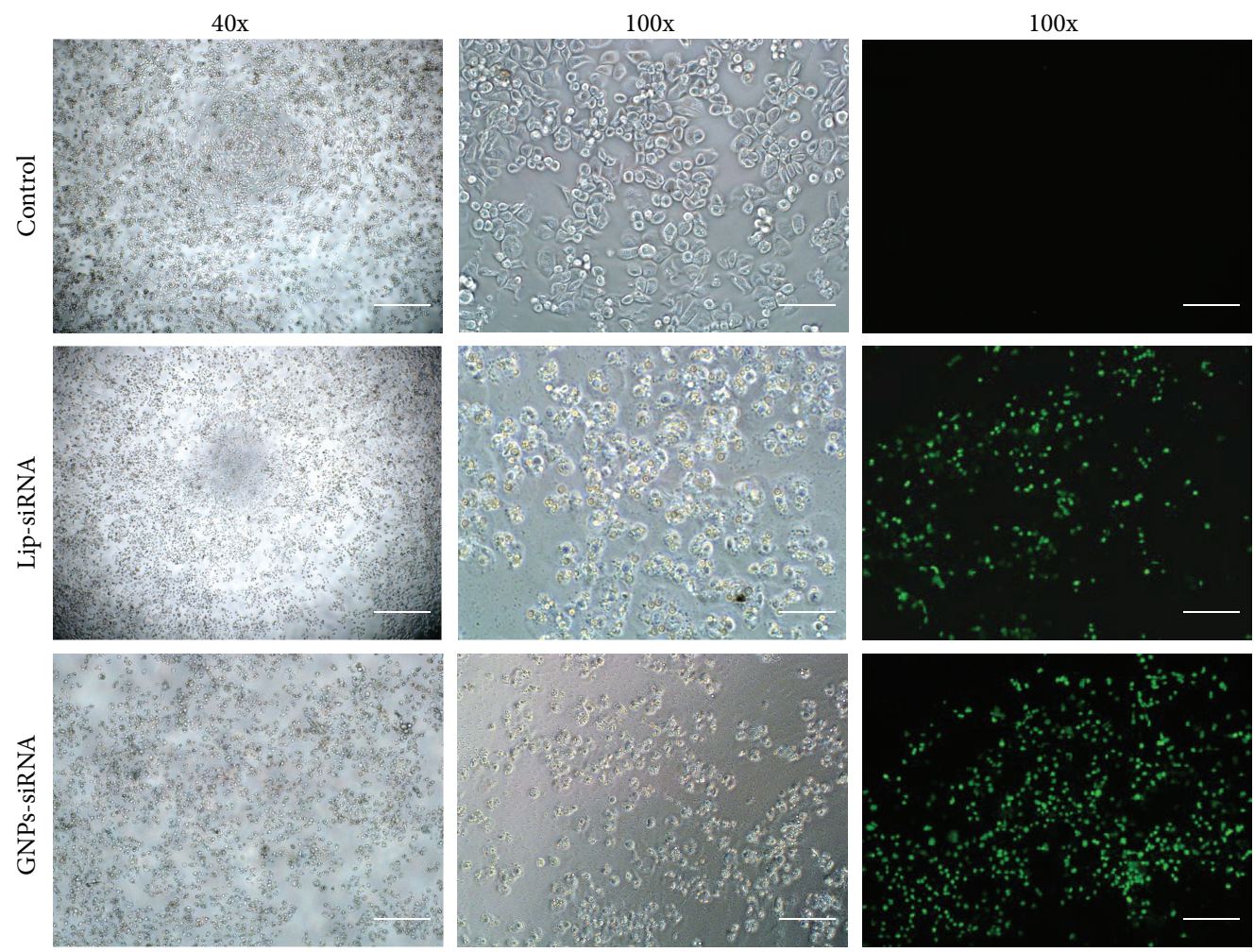

(a)
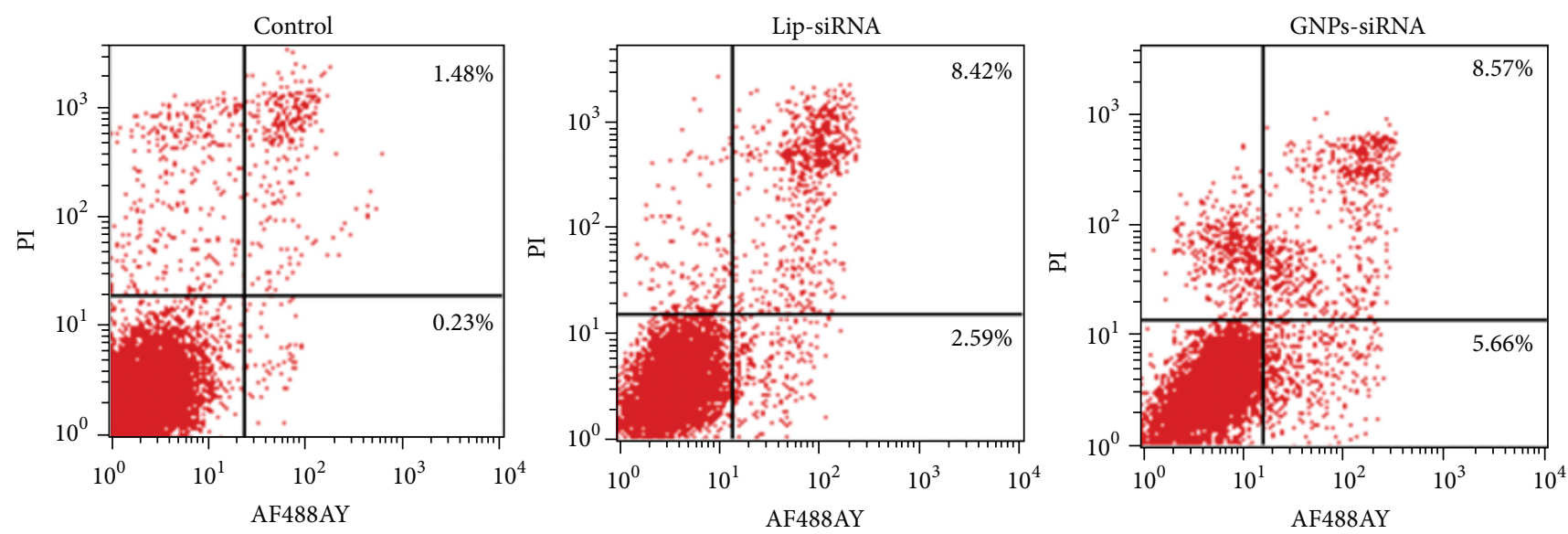

(b)

FIGURE 6: Apoptosis analysis of MCF-7/MDR1 cells after treatment by Lipofectamine 2000-siRNA (Lip-siRNA) and GNPs-siRNA. (a) TUNEL assay. Scale bars: $200 \mu \mathrm{m}(40 \mathrm{x})$ and $100 \mu \mathrm{m}$ (100x). (b) Flow cytometry assay.

and the Natural Science Foundation of Jiangsu Province (BK20130863).

\section{References}

[1] R. Siegel, J. Ma, Z. Zou, and A. Jemal, "Cancer statistics, 2014," CA: A Cancer Journal for Clinicians, vol. 64, no. 1, pp. 9-29, 2014.

[2] L. Fan, K. Strasser-Weippl, J.-J. Li et al., "Breast cancer in China," The Lancet Oncology, vol. 15, no. 7, pp. e279-e289, 2014.
[3] A. Schroeder, D. A. Heller, M. M. Winslow et al., "Treating metastatic cancer with nanotechnology," Nature Reviews Cancer, vol. 12, no. 1, pp. 39-50, 2012.

[4] G. L. David, S. Yegnasubramanian, A. Kumar et al., "MDR1 promoter hypermethylation in MCF-7 human breast cancer cells: changes in chromatin structure induced by treatment with 5-aza-cytidine," Cancer Biology \& Therapy, vol. 3, no. 6, pp. 540$548,2004$.

[5] C. F. Higgins, "Multiple molecular mechanisms for multidrug resistance transporters," Nature, vol. 446, no. 7137, pp. 749-757, 2007. 
[6] D. B. Longley and P. G. Johnston, "Molecular mechanisms of drug resistance," The Journal of Pathology, vol. 205, no. 2, pp. 275-292, 2005.

[7] S. Labialle, L. Gayet, E. Marthinet, D. Rigal, and L. G. Baggetto, "Transcriptional regulation of the human MDR1 gene at the level of the inverted MED-1 promoter region," Annals of the New York Academy of Sciences, vol. 973, pp. 468-471, 2002.

[8] M. M. Gottesman, T. Fojo, and S. E. Bates, "Multidrug resistance in cancer: role of ATP-dependent transporters," Nature Reviews Cancer, vol. 2, no. 1, pp. 48-58, 2002.

[9] B. Song, X. S. Liu, S. J. Rice et al., "Plk1 phosphorylation of Orc2 and Hbol contributes to gemcitabine resistance in pancreatic cancer," Molecular Cancer Therapeutics, vol. 12, no. 1, pp. 58-68, 2013.

[10] K. Strebhardt and A. Ullrich, "Targeting polo-like kinase 1 for cancer therapy," Nature Reviews Cancer, vol. 6, no. 4, pp. 321$330,2006$.

[11] Y. Hikichi, K. Honda, K. Hikami et al., "TAK-960, a novel, orally available, selective inhibitor of polo-like kinase 1, shows broadspectrum preclinical antitumor activity in multiple dosing regimens," Molecular Cancer Therapeutics, vol. 11, no. 3, pp. 700709, 2012.

[12] J. R. Jackson, D. R. Patrick, M. M. Dar, and P. S. Huang, "Targeted anti-mitotic therapies: can we improve on tubulin agents?” Nature Reviews Cancer, vol. 7, no. 2, pp. 107-117, 2007.

[13] E. Herrera-Carrillo and B. Berkhout, "Gene therapy strategies to block HIV-1 replication by RNA interference," in Gene Therapy for HIV and Chronic Infections, vol. 848 of Advances in Experimental Medicine and Biology, pp. 71-95, Springer, New York, NY, USA, 2015.

[14] C. Lei, Y. Cui, L. Zheng, P. Kah-Hoe Chow, and C.-H. Wang, "Development of a gene/drug dual delivery system for brain tumor therapy: potent inhibition via RNA interference and synergistic effects," Biomaterials, vol. 34, no. 30, pp. 7483-7494, 2013.

[15] J. Han, Q. Wang, Z. Zhang, T. Gong, and X. Sun, "Cationic bovine serum albumin based self-assembled nanoparticles as siRNA delivery vector for treating lung metastatic cancer," Small, vol. 10, no. 3, pp. 524-535, 2014.

[16] L. N. Borgheti-Cardoso, L. V. Depieri, H. Diniz et al., "Selfassembling gelling formulation based on a crystalline-phase liquid as a non-viral vector for siRNA delivery," European Journal of Pharmaceutical Sciences, vol. 58, no. 1, pp. 72-82, 2014.

[17] A. Petrelli, R. Carollo, M. Cargnelutti et al., "By promoting cell differentiation, miR-100 sensitizes basal-like breast cancer stem cells to hormonal therapy," Oncotarget, vol. 6, no. 4, pp. 23152330, 2015.

[18] Z. Yang, B. Xiang, D. Dong, Z. Wang, J. Li, and X. Qi, "Dual receptor-specific peptides modified liposomes as VEGF siRNA vector for tumor-targeting therapy," Current Gene Therapy, vol. 14, no. 4, pp. 289-299, 2014.

[19] S. Acharya and R. A. Hill, "High efficacy gold-KDEL peptidesiRNA nanoconstruct-mediated transfection in $\mathrm{C} 2 \mathrm{C} 12$ myoblasts and myotubes," Nanomedicine: Nanotechnology, Biology, and Medicine, vol. 10, no. 2, pp. 329-337, 2014.

[20] D. Heinemann, M. Schomaker, S. Kalies et al., "Gold nanoparticle mediated laser transfection for efficient siRNA mediated gene knock down," PLoS ONE, vol. 8, no. 3, Article ID e58604, 2013.

[21] Y. Tian, J. Sun, H. Yan et al., "A rapid and convenient method for detecting a broad spectrum of malignant cells from malignant pleuroperitoneal effusion of patients using a multifunctional NIR heptamethine dye," Analyst, vol. 140, no. 3, pp. 750-755, 2015.

[22] O. S. Muddineti, B. Ghosh, and S. Biswas, "Current trends in using polymer coated gold nanoparticles for cancer therapy," International Journal of Pharmaceutics, vol. 484, no. 1-2, pp. 252267, 2015.

[23] S. Wang, Z. Teng, P. Huang et al., "Reversibly extracellular $\mathrm{pH}$ controlled cellular uptake and photothermal therapy by pegylated mixed-charge gold nanostars "' Small, vol. 11, no. 15, pp. 1801-1810, 2015.

[24] L. Nie, S. Wang, X. Wang et al., "In vivo volumetric photoacoustic molecular angiography and therapeutic monitoring with targeted plasmonic nanostars," Small, vol. 10, no. 8, pp. 15851593, 2014.

[25] P. Huang, L. Bao, C. Zhang et al., "Folic acid-conjugated silicamodified gold nanorods for X-ray/CT imaging-guided dualmode radiation and photo-thermal therapy," Biomaterials, vol. 32, no. 36, pp. 9796-9809, 2011.

[26] H. Ke, X. Yue, J. Wang et al., "Gold nanoshelled liquid perfluorocarbon nanocapsules for combined dual modal ultrasound/ct imaging and photothermal therapy of cancer," Small, vol. 10, no. 6, pp. 1220-1227, 2014.

[27] Y. Tian, S. Luo, H. Yan et al., "Gold nanostars functionalized with amine-terminated PEG for X-ray/CT imaging and photothermal therapy," Journal of Materials Chemistry B, vol. 3, no. 21, pp. 4330-4337, 2015.

[28] D. Kim, Y. Y. Jeong, and S. Jon, "A drug-loaded aptamergold nanoparticle bioconjugate for combined CT imaging and therapy of prostate cancer," ACS Nano, vol. 4, no. 7, pp. 36893696, 2010. 

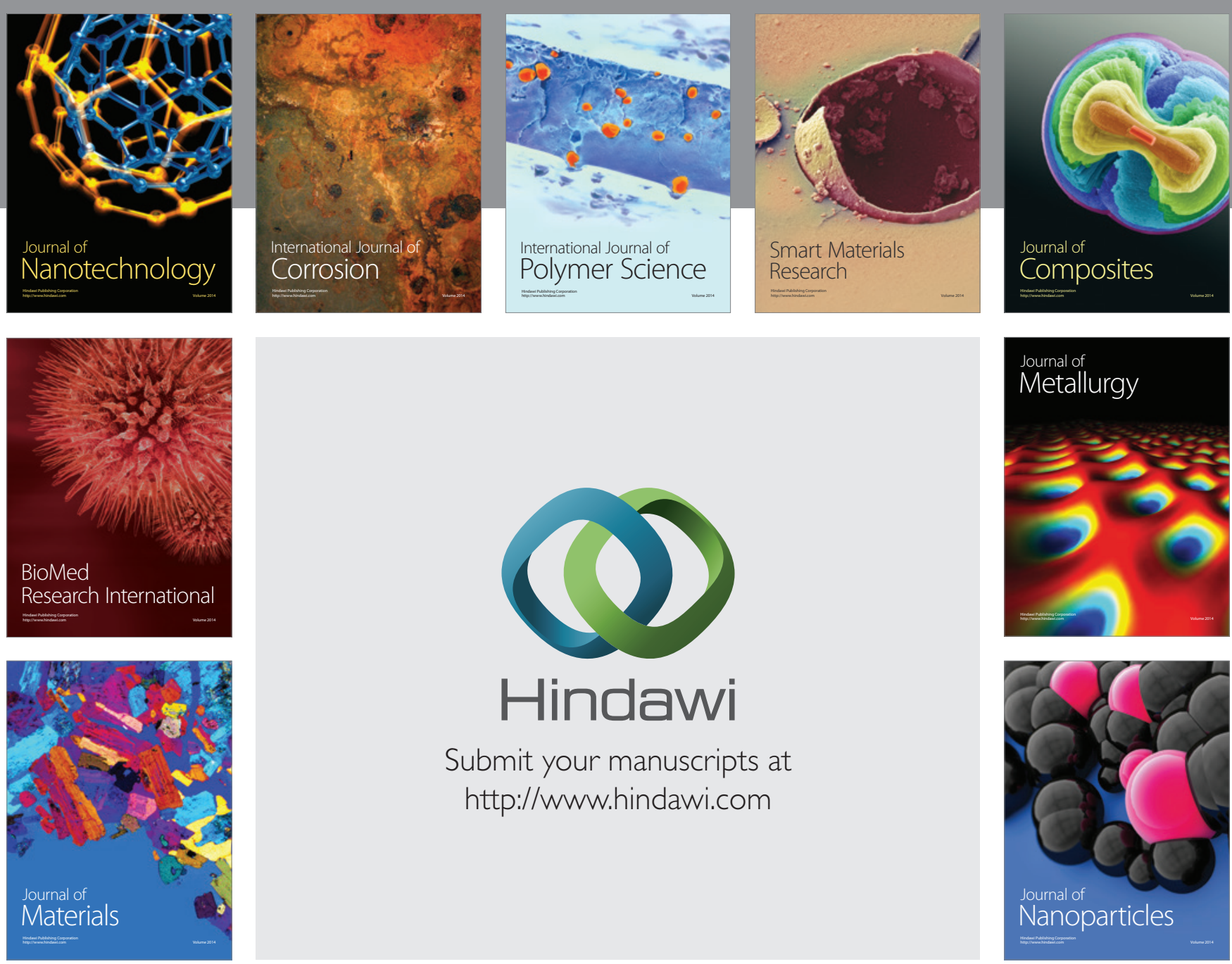

Submit your manuscripts at http://www.hindawi.com
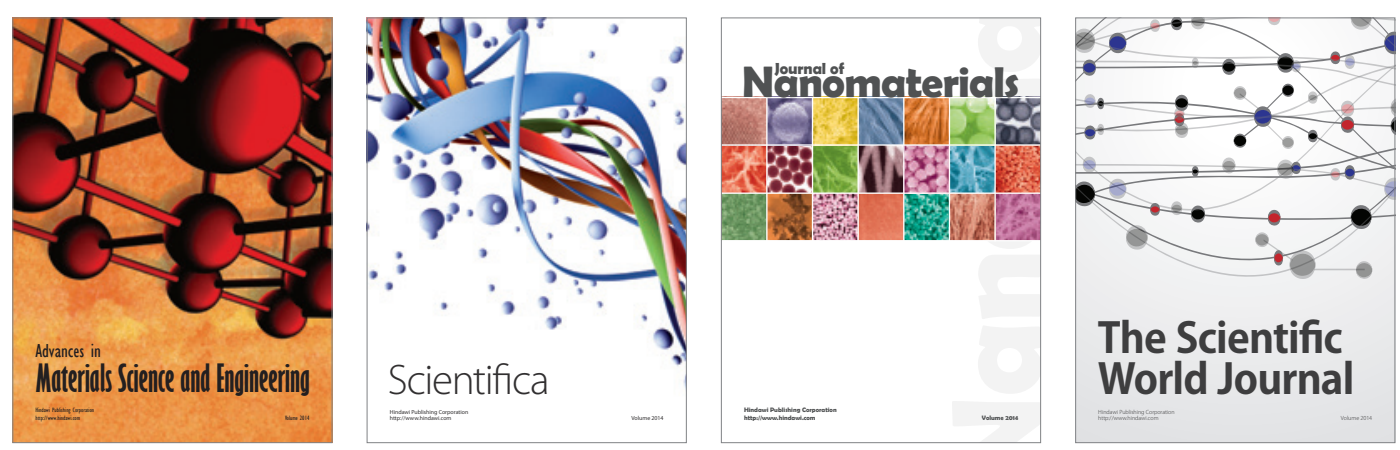

\section{The Scientific World Journal}
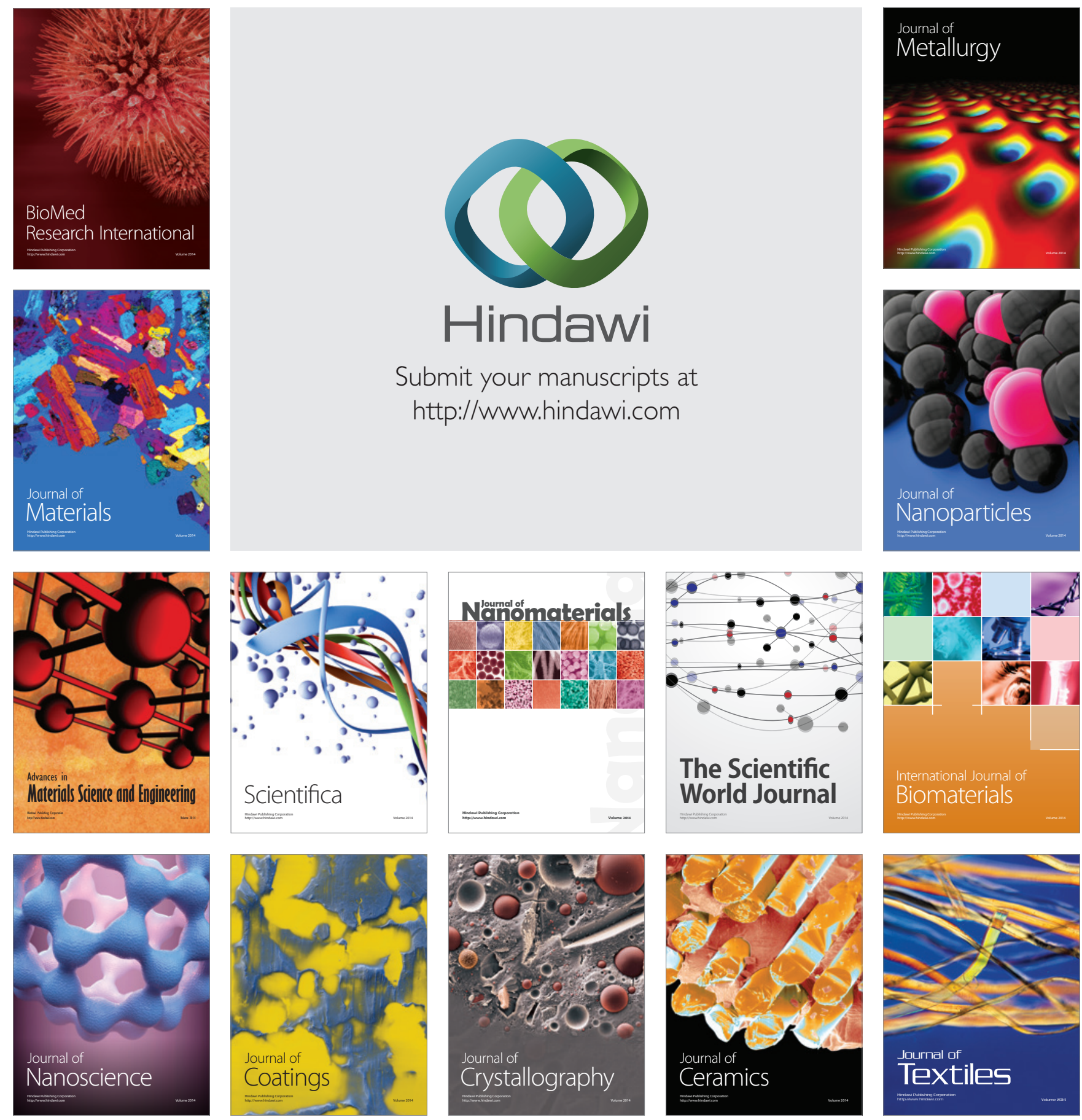